\title{
Use of Tocilizumab in COVID-19: A Systematic Review and Meta-Analysis of Current Evidence
}

Sohny Kotak ${ }^{1}$, Mahima Khatri ${ }^{1}$, Mehreen Malik ${ }^{2}$, Maria Malik ${ }^{3}$, Warda Hassan ${ }^{1}$, Arooba Amjad ${ }^{1}$, Farheen Malik $^{1}$, Hani Hassan ${ }^{4}$, Jawad Ahmed ${ }^{1}$, Marium Zafar ${ }^{5}$

1. Internal Medicine, Dow University of Health Sciences, Karachi, PAK 2. Anesthesiology, Aga Khan University, Karachi, PAK 3. Anesthesiology, Indus Hospital, Lahore, PAK 4. Internal Medicine, Karachi Medical and Dental College, Karachi, PAK 5. Internal Medicine, Dow Medical College, Karachi, PAK

Corresponding author: Jawad Ahmed, jawadahmedd13@gmail.com

\section{Abstract \\ Background and objectives}

A flare-up in coronavirus disease 2019 (COVID-19) cases threatens the health of people, and though there is no proven pharmacological treatment, many analytical studies have suggested that interleukin-6 (IL-6) levels are elevated in cases of severe COVID-19 and that the anti-IL- 6 biologic agent tocilizumab may be beneficial. This is a critical review of studies aiming to assess the safety and efficacy of tocilizumab as compared to the standard regimen in patients with COVID-19.

\section{Materials and methods}

Online databases (PubMed and Cochrane) were searched until June 29, 2020, for original articles investigating the immunological response in COVID-19 and its treatment with tocilizumab. Data on multiple baseline characteristics and pre-specified endpoints were extracted and pooled using a random effect model. We used Review Manager version 5.3 (The Nordic Cochrane Centre, The Cochrane Collaboration, 2014, Denmark) and Stata 11.0 (Stata Corporation LP, College Station, TX) for all analyses. Risk ratios (RR) and the weighted mean difference (WMD) with the corresponding 95\% confidence interval (CI) were calculated.

\section{Results}

From a total of 1,246 identified articles, 13 studies were included after duplicate removal and narrowing based on title and abstract. Of the 13 included studies, seven case-control studies were shortlisted for metaanalysis (quantitative) and six-single arm studies were used in the discussion (qualitative). These studies had 766 patients (351 in the tocilizumab arm and 414 in the control arm). Their pooled analysis demonstrated that mortality was significantly lower in the tocilizumab group $(R R=0.56$ [0.34, 0.92]; $p=0.02$; $\left.\mathrm{I}^{2}=76 \%\right)$, as was the need for artificial invasive ventilation $\left(\mathrm{RR}=0.34[0.12,0.99] ; \mathrm{p}=0.05 ; \mathrm{I}^{2}=0 \%\right)$ as compared to the control group. No significant differences were observed between tocilizumab and control group in intensive care unit admissions $\left(R R=0.73[0.15,3.59] ; p=0.70 ; I^{2}=60 \%\right)$ and risks of post-drug infection

Review began 09/29/2020 Review ended 10/05/2020 Published 10/09/2020

\section{() Copyright 2020}

Kotak et al. This is an open access article distributed under the terms of the Creative Commons Attribution License CC-BY 4.0., which permits unrestricted use, distribution, and reproduction in any medium, provided the original author and source are credited.
$\left(\mathrm{RR}=1.29[0.41,4.04] ; \mathrm{p}=0.66 ; \mathrm{I}^{2}=88 \%\right)$. In terms of efficacy outcome, improved oxygen saturation $(\mathrm{RR}=1.13$

$\left.[1.04,1.65] ; \mathrm{p}=0.02 ; \mathrm{I}^{2}=0 \%\right)$ was reported to be markedly significant in tocilizumab patients when compared with the standard care group.

\section{Conclusions}

Our results based on pooled studies show tocilizumab to be safe and efficacious in reducing mortality among critically ill COVID-19 patients. However, due to the limited number of observational studies, the positive findings should be viewed cautiously and warrant further investigation.

Categories: Internal Medicine, Infectious Disease, Pulmonology

Keywords: covid-19, sars-cov-2, interleukin-6, tocilizumab, systematic review, meta-analysis, coronavirus disease, mortality, safety, efficacy

\section{Introduction}

An exponential increase in the number of patients infected by severe acute respiratory syndrome-related coronavirus (SARS-CoV-2), as well as the rapidly changing disease profile of coronavirus disease 2019 (COVID-19), has been a life-threatening and public health emergency [1]. Despite several disease complications, including gastrointestinal and neurological complications, the leading cause of mortality remains pulmonary failure [2]. Thus, in the quest for developing urgent and effective treatment options for COVID-19 pneumonia, it is crucial to explore treatment options that can slow its progression, reduce the rapid rate of hospitalizations and intensive-care unit (ICU) admissions, reducing the burden on public health systems; and mortality. The current treatment is either supportive or investigational based on 
interim data from studies that are underway.

Although its pathogenesis is still vague, laboratory findings of patients suffering from severe disease show a cytokine storm, involving a considerable release of pro-inflammatory cytokines, among which Interleukin-6 (IL-6) plays a cardinal role [3]. Tocilizumab, a humanized monoclonal antibody, is one of its kind against the IL-6 receptors and may turn down this exaggerated inflammatory response in critically ill patients [4]. Thus, the National Health Commission of China, in its latest 7th version, has advocated the use of tocilizumab in critically ill patients with elevated IL-6 levels [5].

Studies carried out to evaluate the adequacy of tocilizumab so far have yielded different results. Some observational studies showed it to be beneficial, which led to its use in some countries and prompted further research; however, a recent randomized controlled trial (RCT), COVACTA (A Study to Evaluate the Safety and Efficacy of Tocilizumab in Patients With Severe COVID-19 Pneumonia; NCT04320615), suggested no evidence of its benefit in COVID-19 [6]. Hence, the available literature was critically reviewed to find possible reasons for these differences. Additionally, data from different observational studies were pooled and analyzed to report more robust conclusions on the outcomes of tocilizumab in critically ill patients infected with COVID-19 contrasted with the accepted regimen being followed worldwide.

\section{Materials And Methods}

This meta-analysis was conducted according to the guidelines set by Preferred Reporting Items for Systemic Review and Meta-Analysis (PRISMA) and Cochrane guidelines [7-8].

\section{Search strategy}

A systematic literature search was conducted up till June 29, 2020, on the PubMed and Cochrane CENTRAL databases with the following subject keywords and their MeSH terms: ((COVID-19 OR Coronavirus OR SARS-coV-2) AND (Tocilizumab OR IL-6 antagonists OR IL-6 Inhibitors).

Google Scholar and Clinicaltrials.gov were searched for any studies that had not yet been published but had reported their results online. There was no language barrier, as all the studies retrieved in the search were in the English language. Two reviewers independently screened the search results. A third reviewer was consulted in case of discrepancies. Duplicates were removed and studies were initially shortlisted based on title and abstract, after which the full text was assessed for eligibility. References of the selected studies were also reviewed thoroughly to prevent any risk of selection bias.

\section{Inclusion and exclusion criteria}

Observational studies, including adults $\geqslant 18$ years with polymerase chain reaction (PCR)-confirmed SARSCoV-2 infection in which the efficacy of tocilizumab added to standard therapy and standard therapy alone, were compared. Additionally, studies that had published full-text in the English language were considered. Only articles were excluded if they were reviews, editorials, or case reports. All identified studies were imported in EndNote Reference Library version X4 (Clarivate Analytics, Thomson Reuters Corporation, Philadelphia, Pennsylvania) for the removal of duplicates.

\section{Data extraction}

Data extraction of the relevant studies included the first author, year of publication, type of study, study follow-up time, total number of COVID-19 positive patients, and patients who received tocilizumab. From the obtained relevant studies, the baseline characteristics (such as age, gender, and standard therapy) and comorbidities (such as heart disease, hypertension, diabetes mellitus, etc.) of patients in the two groups were also extracted. We accepted the study investigator's definition for all safety and efficacy outcomes. The outcomes extracted are mentioned below.

\section{Safety outcomes}

Safety outcomes included mortality, ICU admission, risk of infection (bacteremia, fungemia, and candidiasis), need for mechanical ventilation, and serious adverse events.

\section{Efficacy outcome}

Improved oxygen saturation was considered an efficacy outcome.

\section{Assessment of risk of bias}

Quality assessment of all the observational studies was done using the Newcastle-Ottawa reference scale [9].

\section{Statistical analysis}

Statistical analysis was done only for comparative studies using Review Manager version 5.3 (The Nordic 
Cochrane Centre, The Cochrane Collaboration, 2014, Denmark) and Stata 11.0 (Stata Corporation LP, College Station, TX). Relative risks (RR) with 95\% confidence intervals (CIs) were calculated using raw study data and were pooled using a random-effects model for dichotomous data. Due to the lack of adjusted outcome measures, baseline differences were assessed between the tocilizumab and control group patients in this study to identify potential confounding factors. Funnel plots for all outcomes were visualized and Begg's test was performed to assess publication bias. Potential causes of heterogeneity were explored by carrying out a subgroup analysis according to the use of corticosteroids analyses. The Higgins $\mathrm{I}^{2}$ statistic was used to evaluate heterogeneity, and the value of $25 \%-50 \%$ as mild, $50 \%-75 \%$ as moderate, and $>75 \%$ was considered significant heterogeneity. A p-value of $\leqslant 0.05$ was considered significant.

\section{Results}

A total of 1,246 articles were identified initially from the literature search, 1,231 from database searches and 15 from other sources such as references of relevant studies. Finally, 13 retrospective observational studies were found, amongst which seven were comparative [10-16] and six were single-arm [17-22]. In this meta-analysis, only seven comparative studies were included. A detailed search is illustrated in the PRISMA flow-chart (Figure 1).

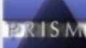

PRISMA 2009 Flow Diagram
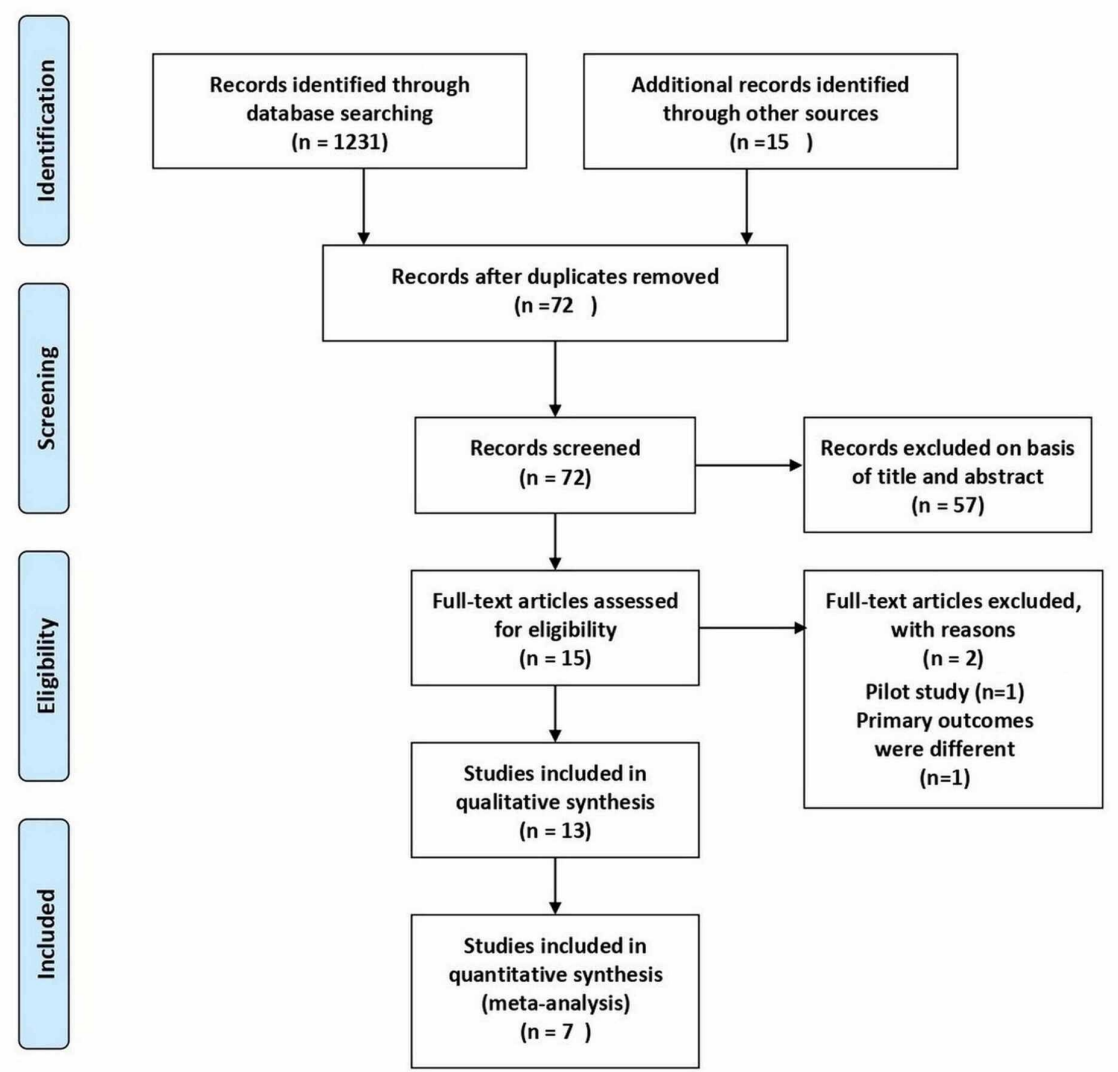

\section{FIGURE 1: PRISMA flow chart for study selection}

PRISMA: Preferred Reporting Items for Systemic Review and Meta-Analysis

\section{Quality assessment and publication bias}

The quality assessment of studies using the New Castle Ottawa scale depicted a significantly low risk of bias in all the included case-control studies (Table 1). The funnel plots showed no publication bias (Figure 2), which was confirmed by Begg's test with the exception of improved oxygen saturation (efficacy outcome). The details of Begg's test for all outcomes is given in Table 2. 


\section{Cureus}

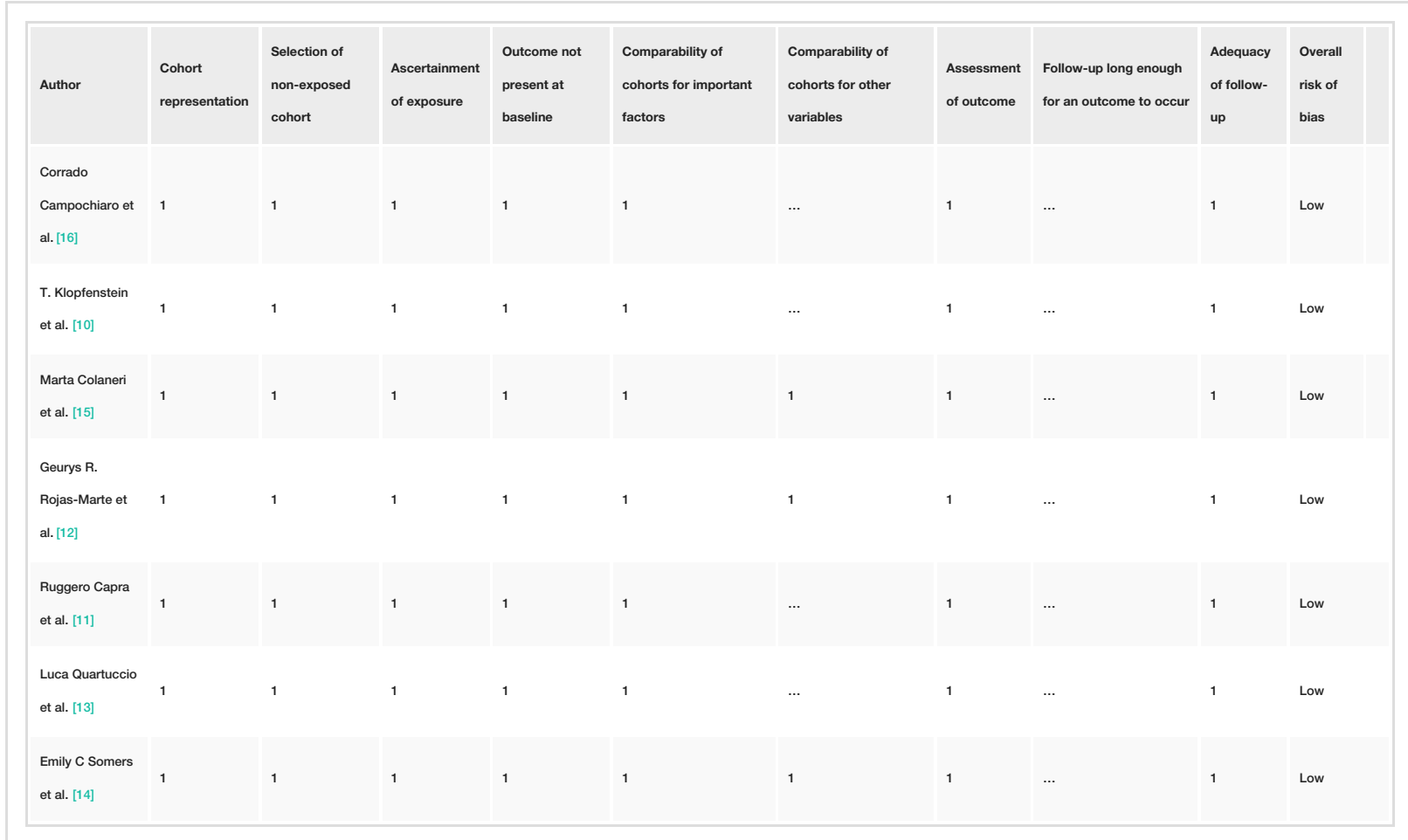

TABLE 1: Quality assessment of the included observational studies

A

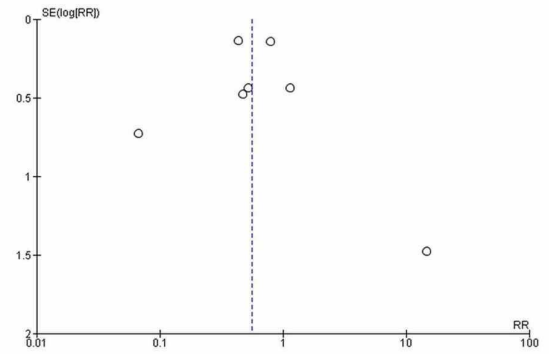

C

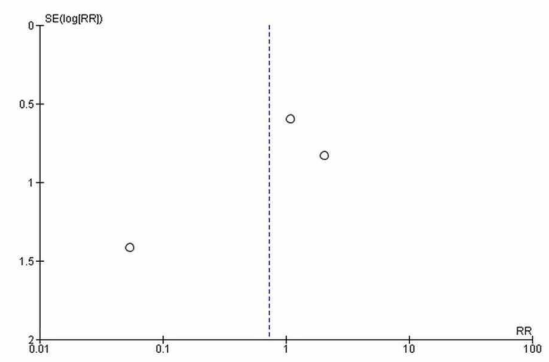

E

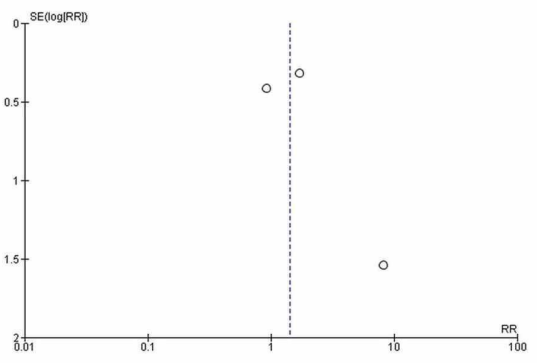

B

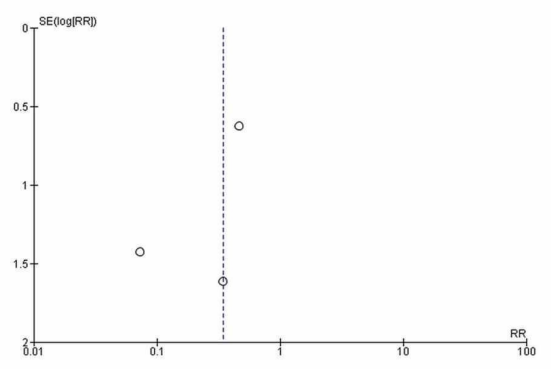

D

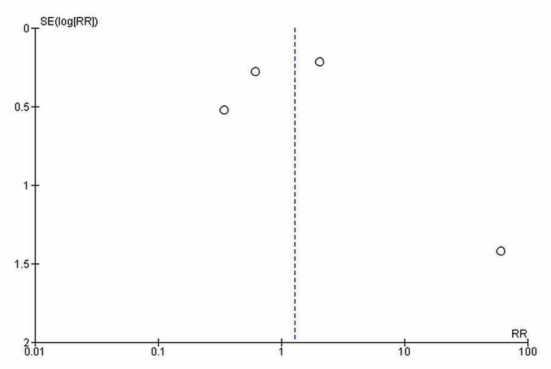

$\mathbf{F}$

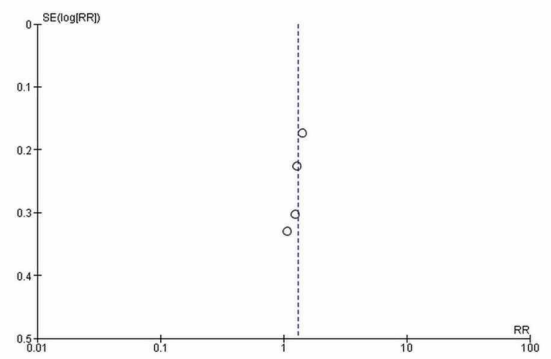




\section{Cureus}

\section{FIGURE 2: Funnel plots for publication bias}

(A) Mortality; (B) Post-therapy mechanical ventilation; (C) ICU admission; (D) Frequency of post-drug infection; (E) Serious adverse events; and (F) Improved oxygenation.

SE, standard error; RR, risk ratio; ICU, intensive care unit

\begin{tabular}{|c|c|c|}
\hline Category & Outcomes & Begg's p-value \\
\hline \multirow{5}{*}{ Safety outcomes } & Mortality & 0.881 \\
\hline & Post-therapy mechanical ventilation & 0.317 \\
\hline & ICU admission & 0.317 \\
\hline & Post-drug infection & 1.000 \\
\hline & Serious adverse events & 0.602 \\
\hline Efficacy outcomes & Improved oxygen saturation & 0.042 \\
\hline
\end{tabular}

TABLE 2: Results of Begg's test of publication bias for all outcomes

\section{Baseline characteristics}

The seven studies included 766 patients (351 in the tocilizumab arm and 414 in the control arm). The baseline characteristics and comorbidities of the comparative studies are given in Table 3 and Table 4 , respectively. 


\section{Cureus}

\begin{tabular}{|c|c|c|c|c|c|c|c|c|c|}
\hline \multirow[t]{2}{*}{ Study } & \multirow[t]{2}{*}{ Type of study } & \multirow[t]{2}{*}{$\begin{array}{l}\text { Follow- } \\
\text { up } \\
\text { period } \\
\text { (days) }\end{array}$} & \multirow[t]{2}{*}{$\begin{array}{l}\text { Total } \\
\text { No. } \\
\text { of } \\
\text { Pts }\end{array}$} & \multirow[t]{2}{*}{$\begin{array}{l}\text { Patients } \\
\text { receiving } \\
\text { tocilizumab } \\
\text { (n) }\end{array}$} & \multicolumn{2}{|l|}{ Male (n) } & \multicolumn{2}{|l|}{ Age (years) } & \multirow[t]{2}{*}{ Standard therapy } \\
\hline & & & & & $\begin{array}{l}\text { Tocilizumab } \\
\text { group }\end{array}$ & $\begin{array}{l}\text { Control } \\
\text { group }\end{array}$ & $\begin{array}{l}\text { Tocilizumab } \\
\text { group }\end{array}$ & $\begin{array}{l}\text { Control } \\
\text { group }\end{array}$ & \\
\hline $\begin{array}{l}\text { Rojas-Marte } \\
\text { et al. [12] }\end{array}$ & $\begin{array}{l}\text { Retrospective, } \\
\text { case-control, } \\
\text { single-center } \\
\text { study }\end{array}$ & 48 & 193 & 96 & 74 & 63 & $58.8 \pm 13.6$ & $\begin{array}{l}62.0 \pm \\
14\end{array}$ & $\begin{array}{l}\text { Hydroxychloroquine, azithromycin, corticosteroids, } \\
\text { remdesivir, and anticoagulation }\end{array}$ \\
\hline $\begin{array}{l}\text { Marta } \\
\text { Colaneri et al. } \\
{[15]}\end{array}$ & $\begin{array}{l}\text { Retrospective, } \\
\text { single-center }\end{array}$ & 7 & 112 & 21 & 19 & 63 & $\begin{array}{l}62.33 \pm \\
18.68\end{array}$ & $\begin{array}{l}63.74 \pm \\
16.32\end{array}$ & $\begin{array}{l}\text { Combination of hydroxychloroquine ( } 200 \mathrm{mg} \text { bid), } \\
\text { azithromycin ( } 500 \mathrm{mg} \text { once), a prophylactic dose of low } \\
\text { weight heparin, and methylprednisolone (a tapered } \\
\text { dose of } 1 \mathrm{mg} / \mathrm{kg} \text { up to a maximum of } 80 \mathrm{mg} \text { ) for } 10 \\
\text { days. }\end{array}$ \\
\hline $\begin{array}{l}\text { Klopfenstein } \\
\text { et al. [10] }\end{array}$ & $\begin{array}{l}\text { Retrospective, } \\
\text { case-control, } \\
\text { single-center } \\
\text { study }\end{array}$ & 13 & 45 & 20 & NR & NR - & $76.8 \pm 11$ & $70.7 \pm 15$ & $\begin{array}{l}\text { Hydroxychloroquine or lopinavir-ritonavir therapy and } \\
\text { antibiotics, and less commonly corticosteroids }\end{array}$ \\
\hline $\begin{array}{l}\text { Corrado } \\
\text { Campochiaro } \\
\text { et al. [16] }\end{array}$ & $\begin{array}{l}\text { Retrospective, } \\
\text { case-control, } \\
\text { single-center } \\
\text { study }\end{array}$ & 28 & 65 & 32 & 29 & 27 & $64(53-75)$ & $\begin{array}{l}60(55- \\
75.5)\end{array}$ & $\begin{array}{l}\text { Hydroxychloroquine } 400 \mathrm{mg} \text {, daily, lopinavir/ritonavir } \\
400 / 100 \mathrm{mg} \text { twice daily, ceftriaxone } 2 \text { gr for } 6 \text { days, } \\
\text { azithromycin } 500 \mathrm{mg} \text { daily until a negative report of } \\
\text { urine. enoxaparin } 4000 \mathrm{IU} \text { subcutaneously once a day }\end{array}$ \\
\hline $\begin{array}{l}\text { Ruggero } \\
\text { Capra et al. } \\
\text { [11] }\end{array}$ & Retrospective & 21 & 85 & 62 & 45 & 19 & $63(54-73)$ & $\begin{array}{l}70(55- \\
80)\end{array}$ & $\begin{array}{l}\text { Hydroxychloroquine } 400 \mathrm{mg} \text { daily and lopinavir } 800 \mathrm{mg} \\
\text { daily plus ritonavir } 200 \mathrm{mg} \text { daily }\end{array}$ \\
\hline $\begin{array}{l}\text { Luca } \\
\text { Quartuccio et } \\
\text { al. [13] }\end{array}$ & $\begin{array}{l}\text { Single-center } \\
\text { retrospective } \\
\text { study }\end{array}$ & 38 & 111 & 42 & 33 & 44 & $62.4 \pm 11.8$ & $\begin{array}{l}56.2 \pm \\
14.2\end{array}$ & $\begin{array}{l}\text { Lopinavir/ritonavir, darunavir/cobicistat, remdesivir, } \\
\text { hydroxychloroquine, chloroquine, and } \\
\text { methylprednisolone }\end{array}$ \\
\hline $\begin{array}{l}\text { Emily C } \\
\text { Somers et } \\
\text { al. [14] }\end{array}$ & $\begin{array}{l}\text { Single-center } \\
\text { retrospective } \\
\text { study }\end{array}$ & 47 & 154 & 78 & 53 & 49 & $55 \pm 14.9$ & $60 \pm 14.5$ & $\begin{array}{l}\text { Hydroxychloroquine, remdesivir, NSAIDs, } \\
\text { acetaminophen, ace inhibitors or angiotensin receptor } \\
\text { blockers, vasopressors, anticoagulants, oral } \\
\text { prednisone, and methylprednisolone }\end{array}$ \\
\hline
\end{tabular}

TABLE 3: Baseline characteristics of the case-control studies included in the meta-analysis bid, twice a day; IU, international units 


\section{Cureus}

\begin{tabular}{|c|c|c|c|c|c|c|c|c|}
\hline \multirow{2}{*}{ Study } & \multicolumn{2}{|c|}{ Heart disease $n(\%)$} & \multicolumn{2}{|c|}{ Hypertension n (\%) } & \multicolumn{2}{|c|}{ COPD n (\%) } & \multicolumn{2}{|c|}{ Diabetes mellitus $\mathrm{n}(\%)$} \\
\hline & $\begin{array}{l}\text { TCZ } \\
\text { group }\end{array}$ & $\begin{array}{l}\text { Control } \\
\text { group }\end{array}$ & $\begin{array}{l}\text { TCZ } \\
\text { group }\end{array}$ & $\begin{array}{l}\text { Control } \\
\text { group }\end{array}$ & $\begin{array}{l}\text { TCZ } \\
\text { group }\end{array}$ & $\begin{array}{l}\text { Control } \\
\text { group }\end{array}$ & $\begin{array}{l}\text { TCZ } \\
\text { group }\end{array}$ & $\begin{array}{l}\text { Control } \\
\text { group }\end{array}$ \\
\hline Rojas-Marte et al. [12] & $11(11.5)$ & $18(18.5)$ & $53(55.2)$ & $51(52.6)$ & $8(8.3)$ & $3(3.1)$ & $29(30.2)$ & $38(39.2)$ \\
\hline Marta Colaneri et al. [15] & $2(9.5)$ & $7(7.7)$ & $8(38)$ & $20(21.9)$ & 0 & $4(19)$ & $2(9.5)$ & $8(8.8)$ \\
\hline Klopfenstein et al. [10] & $14(70)$ & $17(68)$ & $11(55)$ & $11(44)$ & $4(20)$ & $1(4)$ & $5(25)$ & $8(32)$ \\
\hline $\begin{array}{l}\text { Corrado Campochiaro et al. } \\
\text { [16] }\end{array}$ & $4(12)$ & $6(18)$ & $12(37)$ & $16(48)$ & $1(3)$ & $2(6)$ & $4(12)$ & $6(18)$ \\
\hline Ruggero Capra et al. [11] & $8(14)$ & $6(26)$ & $28(46)$ & $11(488)$ & 88 & 88 & $88(14)$ & $5(22)$ \\
\hline Luca Quartuccio et al. [13] & NR & NR & $20(47.6)$ & $21(30.4)$ & NR & NR & NR & NR \\
\hline Emily C Somers et al. [14] & $16(21)$ & $20(26)$ & $50(64)$ & $52(68)$ & $8(10)$ & $21(28)$ & $10(13)$ & $15(20)$ \\
\hline
\end{tabular}

\section{TABLE 4: Comorbidities of the case-control studies included in the meta-analysis}

TCZ, tocilizumab; COPD, chronic obstructive pulmonary disease; NR, not reported

\section{Assessment of baseline differences}

When baseline characteristics were pooled, we found significantly more male patients $(\mathrm{RR}=1.13[1.02,1.26]$; $\mathrm{p}=0.02)$ and less diabetic patients $(\mathrm{RR}=0.74[0.55,0.99] ; \mathrm{p}=0.04)$ in the tocilizumab group. All the remaining characteristics were insignificant between both the intervention groups. Differences in key baseline characteristics between the tocilizumab group and the control group are represented in Table 5 . The forest plots for the baseline assessment difference are given in Figure 3.

\begin{tabular}{|c|c|c|}
\hline Baseline characteristics & Tocilizumab vs. control patients $[95 \% \mathrm{Cl}]$ & p-value \\
\hline Age & $W M D=-0.29[-4.48,4.10]$ & 0.90 \\
\hline Males & $\mathrm{RR}=1.13[1.02,1.26]$ & 0.02 \\
\hline CRP levels (mg/dL) & WMD $=-2.26[-17.21,12.70]$ & 0.77 \\
\hline Hypertension & $R R=1.06[0.90,1.26]$ & 0.47 \\
\hline Heart disease & $\mathrm{RR}=0.83[0.64,1.09]$ & 0.18 \\
\hline COPD & $\mathrm{RR}=1.02[0.31,3.31]$ & 0.98 \\
\hline Diabetes mellitus & $R R=0.74[0.55,0.99]$ & 0.04 \\
\hline
\end{tabular}

TABLE 5: Pooled baseline demographics comparing tocilizumab group and control group patients CRP, C-reactive protein; COPD, chronic obstructive pulmonary disease; RR, relative risks; WMD, weighted mean difference; $\mathrm{Cl}$, confidence interval 


\section{Cureus}

A.

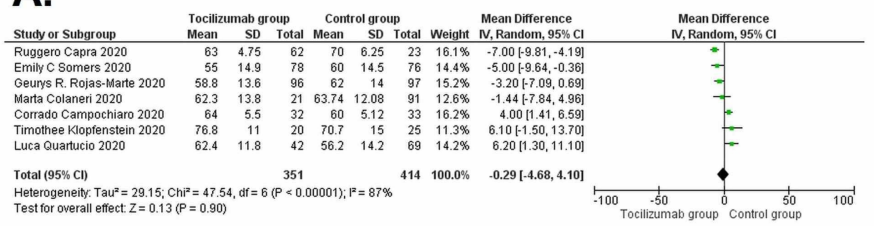

B.

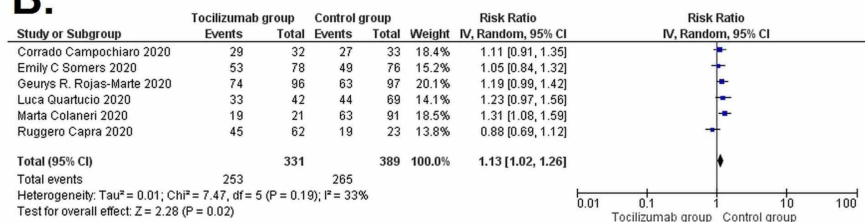

C.

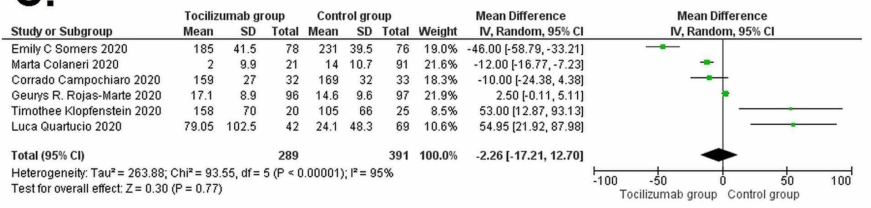

D.

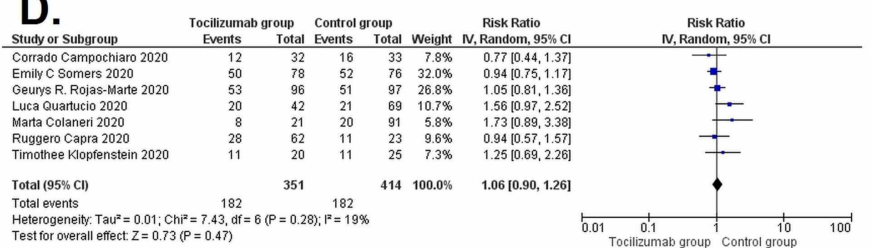

E.

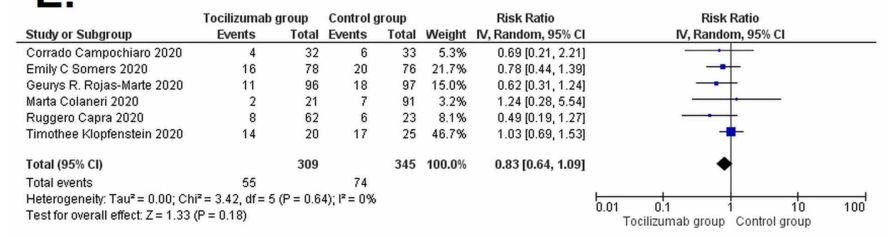

F.

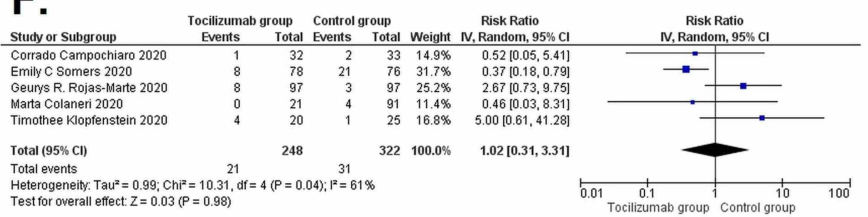

G.

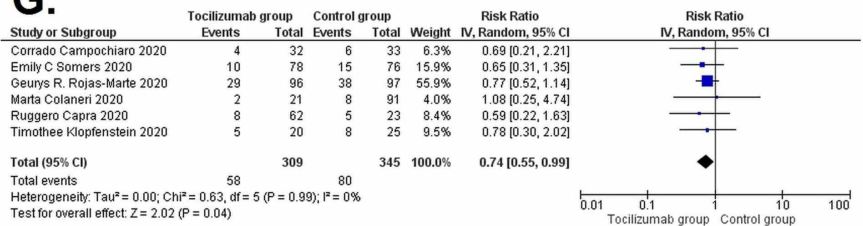

FIGURE 3: Forest plots showing pooled baseline demographics comparing tocilizumab group and control group patients

(A) Age; (B) Males; (C) C-reactive protein levels; (D) Hypertension; (E) Heart disease; (F) Chronic obstructive pulmonary disease; and (G) Diabetes mellitus

\section{Safety outcomes}

The pooled analysis of the patients receiving tocilizumab showed a significantly lower risk of mortality $\left(\mathrm{RR}=0.56[0.34,0.92] ; \mathrm{p}=0.02 ; \mathrm{I}^{2}=76 \%\right.$ ) as compared to the control group, and no publication bias was found for the mortality outcome $(\mathrm{p}=0.20)$. Also, there was a significant decrease in patients needing post-therapy mechanical ventilation $\left(\mathrm{RR}=0.34[0.12,0.99], \mathrm{p}=0.05, \mathrm{I}^{2}=0 \%\right.$ ) in the tocilizumab group versus the control group. Yet, no significant differences were observed between the two groups in terms of ICU admissions $\left(R R=0.73[0.15,3.59] ; p=0.70, I^{2}=60 \%\right)$, risk of post-drug infection $\left(R R=1.29[0.41,4.04] ; p=0.66 ; I^{2}=88 \%\right)$ and 


\section{Cureus}

serious adverse events $\left(\mathrm{RR}=1.42[0.75,2.71] ; \mathrm{p}=0.28 ; \mathrm{I}^{2}=27 \%\right)$. The forest plots for all the above-mentioned outcomes are displayed in Figure 4.

A.

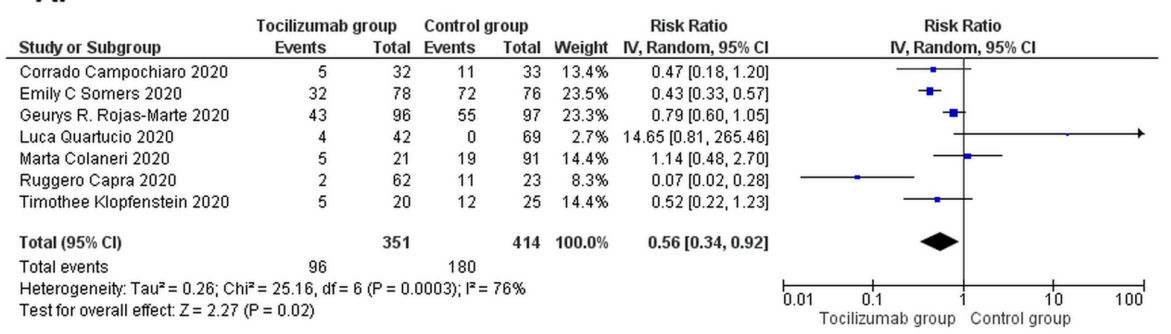

B.

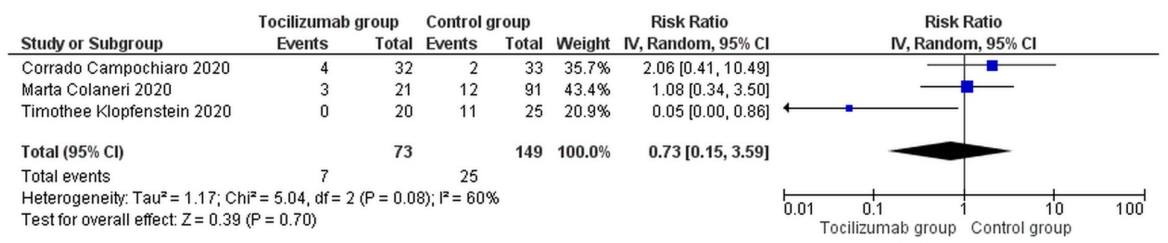

c.

\begin{tabular}{|c|c|c|c|c|c|c|c|c|}
\hline \multirow[b]{2}{*}{ Study or Subgroup } & \multicolumn{2}{|c|}{ Tocilizumab group } & \multicolumn{2}{|c|}{ Control group } & \multirow{2}{*}{ Weight } & \multirow{2}{*}{$\begin{array}{c}\text { Risk Ratio } \\
\text { IV, Random, } 95 \% \mathrm{Cl}\end{array}$} & \multirow{2}{*}{\multicolumn{2}{|c|}{$\begin{array}{c}\text { Risk Ratio } \\
\text { IV, Random, } 95 \% \mathrm{Cl}\end{array}$}} \\
\hline & Events & Total & Events & Total & & & & \\
\hline Corrado Campochiaro 2020 & 0 & 32 & 1 & 33 & $11.2 \%$ & $0.34[0.01,8.13]$ & & \\
\hline Ruggero Capra 2020 & 5 & 62 & 4 & 23 & $74.5 \%$ & $0.46[0.14,1.58]$ & & \\
\hline Timothee Klopfenstein 2020 & 0 & 20 & 8 & 25 & $14.3 \%$ & $0.07[0.00,1.19]$ & & \\
\hline Total $(95 \% \mathrm{Cl})$ & & 114 & & 81 & $100.0 \%$ & $0.34[0.12,0.99]$ & & \\
\hline \multirow{2}{*}{\multicolumn{7}{|c|}{$\begin{array}{l}\text { Heterogeneity: Tau" }=0.00 ; \mathrm{Ch}^{2}=1.41, \mathrm{df}=2(P=0.49) ; \mathrm{I}^{2}=0 \% \\
\text { Test for overall effect: } Z=1.98(P=0.05)\end{array}$}} & & \\
\hline & & & & & & & ${ }^{0.01} \frac{0.1}{\text { Tocilizumab group }^{1}}$ & ${ }^{1} \begin{array}{c}10 \\
\text { Control group }\end{array}$ \\
\hline
\end{tabular}

D.

\begin{tabular}{|c|c|c|c|c|c|c|c|c|c|}
\hline Study or Subgroup & \multicolumn{2}{|c|}{ Tocilizumab group } & \multicolumn{2}{|c|}{ Control group } & Weight & $\begin{array}{c}\text { Risk Ratio } \\
\text { IV, Random, } 95 \% \mathrm{Cl} \\
\end{array}$ & \multicolumn{3}{|c|}{$\begin{array}{c}\text { Risk Ratio } \\
\text { IV, Random, } 95 \% \mathrm{Cl} \\
\end{array}$} \\
\hline Corrado Campochiaro 2020 & 4 & 32 & 12 & 33 & $26.2 \%$ & $0.34[0.12,0.95]$ & & & \\
\hline Emily C Somers 2020 & 42 & 78 & 20 & 76 & $31.8 \%$ & $2.05[1.33,3.14]$ & & $\rightarrow$ & \\
\hline Geurys R. Rojas-Marte 2020 & 16 & 96 & 26 & 97 & $30.8 \%$ & $0.62[0.36,1.08]$ & & & \\
\hline Luca Quartucio 2020 & 18 & 42 & 0 & 69 & $11.1 \%$ & $60.23[3.72,974.03]$ & & & \\
\hline Total $(95 \% \mathrm{Cl})$ & & 248 & & 275 & $100.0 \%$ & $1.29[0.41,4.04]$ & & & \\
\hline Total events & 80 & & 58 & & & & & & \\
\hline $\begin{array}{l}\text { Heterogeneity: Tau }{ }^{2}=1.01 ; \mathrm{Ch} \\
\text { Test for overall effect: } Z=0.44\end{array}$ & $\begin{array}{l}z=24.69, \mathrm{df} \\
P=0.66)\end{array}$ & $(P<0.0$ & 0001); $F^{2}=$ & & & & ${ }^{0.01} \begin{array}{c}0.1 \\
\text { Tocilizumab aroup }\end{array}$ & $\begin{array}{r}10 \\
\text { Control aroup }\end{array}$ & 100 \\
\hline
\end{tabular}

E.

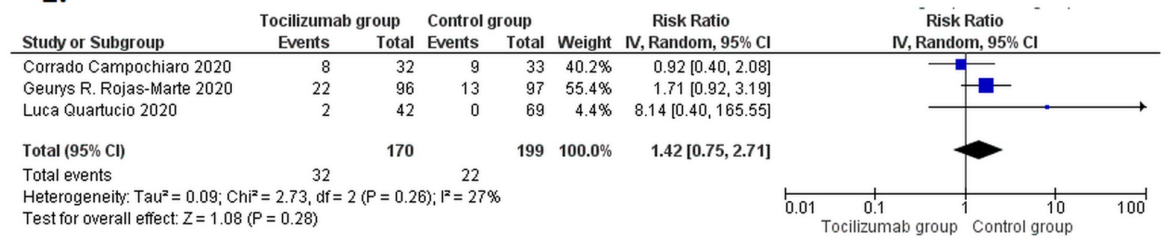

\section{FIGURE 4: Forest plots for safety outcomes}

(A) Mortality; (B) Intensive care unit admission; (C) Post-therapy mechanical ventilation; (D) Post-drug infection; and (E) Serious adverse events

CI, confidence interval; IV, inverse variance; M-H, Mantel-Haenszel

The studies used in the analysis include [10-16].

\section{Efficacy outcome}

However, in terms of efficacy outcome, improved oxygen saturation (RR=1.13 [1.04, 1.65]; $\mathrm{p}=0.02 ; \mathrm{I}^{2}=0 \%$ ) was reported to be markedly significant in tocilizumab patients when compared with the standard care group as shown in Figure 5. 


\section{Cureus}

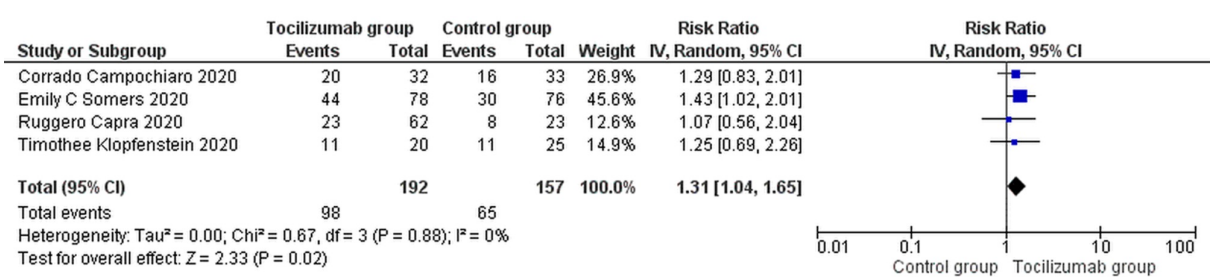

FIGURE 5: Forest plots for efficacy outcome (improved oxygenation)

$\mathrm{Cl}$, confidence interval; IV, inverse variance; $\mathrm{M}-\mathrm{H}$, Mantel-Haenszel

Studies used in the analysis include $[10-11,14,16]$

\section{Subgroup analysis by corticosteroid use}

Subgroup analysis was performed to check whether the administration of corticosteroids in addition to tocilizumab and standard care influenced the results produced, and no significant difference was found in any safety and efficacy outcome among the subgroups except that infection was markedly reduced in those patients who were given corticosteroids ( $\mathrm{RR}=1.29$ [0.41, 4.04]; $\mathrm{p}=0.04 ; \mathrm{I}^{2}=77.5 \%$ ). The details of other subgroup analyses are given in Table 6 and individual forest plots are given in Figure 6 .

\begin{tabular}{|c|c|c|c|c|c|}
\hline \multicolumn{2}{|l|}{ Outcomes } & $\begin{array}{l}\text { Corticosteroids given } \\
\text { RR }(95 \% \mathrm{Cl})\end{array}$ & $\begin{array}{l}\text { No corticosteroids given } \\
\text { RR }(95 \% \mathrm{Cl})\end{array}$ & $P_{\text {subgroups }}$ & $I^{2}(\%)$ \\
\hline \multirow{5}{*}{ Safety outcomes } & Mortality & $0.70(0.43,1.15)$ & $0.19(0.03,1.28)$ & 0.2 & 40.4 \\
\hline & Intensive care unit admission & $0.32(0.02,5.73)$ & $2.06(0.41,10.49)$ & 0.27 & 18.1 \\
\hline & Post-therapy mechanical ventilation & $0.07(0.00,1.19)$ & $0.45(0.14,1.40)$ & 0.24 & 27.8 \\
\hline & Post drug infection & $2.06(0.55,7.66)$ & $0.34(0.12,0.95)$ & 0.04 & 77.5 \\
\hline & Serious adverse events & $1.82(0.99,3.36)$ & $0.92(0.40,2.08)$ & 0.19 & 42.5 \\
\hline Efficacy outcome & Improved oxygen saturation & $1.38(1.03,1.86)$ & $1.21(0.84,1.75)$ & 0.59 & 0 \\
\hline
\end{tabular}

TABLE 6: Subgroup analysis by corticosteroid use for safety and efficacy outcomes

$\mathrm{RR}$, Relative risk; $\mathrm{Cl}$, Confidence Interval; $\mathrm{p}_{\text {subgroups }}, \mathrm{p}$-value for subgroup differences 


\section{Cureus}

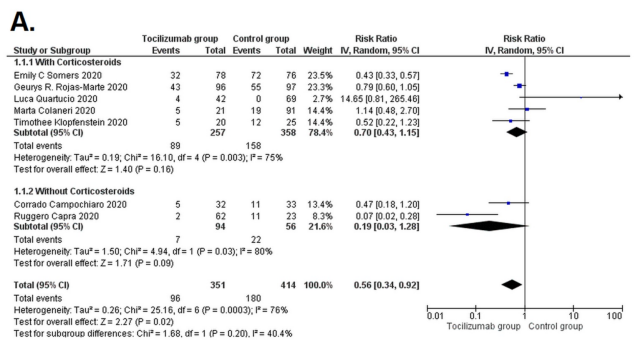

B.

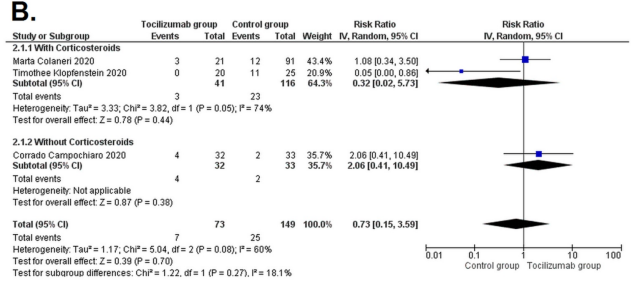

C.

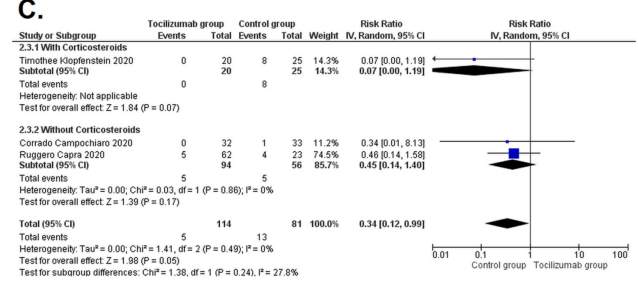

D.

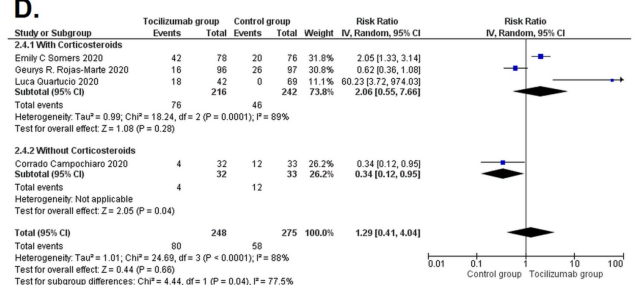

E.

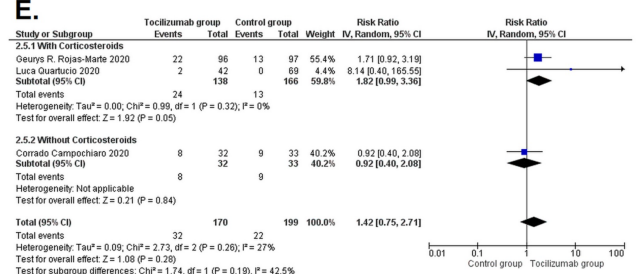

F.

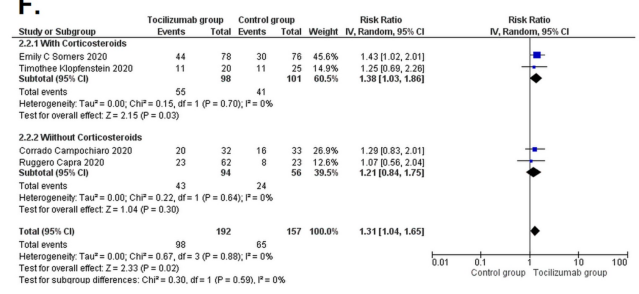

FIGURE 6: Forest plots showing subgroup analysis by corticosteroid use for all outcomes

(A) Mortality; (B) Intensive care unit admission; (C) Post-therapy mechanical ventilation; (D) Post-drug infection; (E) Serious adverse events; and (F) Improved oxygen saturation.

$\mathrm{Cl}$, confidence interval; IV, inverse variance; $\mathrm{M}-\mathrm{H}$, Mantel-Haenszel

Studies used in the analysis include [10-16].

\section{Discussion}

Our meta-analysis of seven comparative observational studies, in summary, demonstrated two key findings. Firstly, tocilizumab was superior in lowering mortality rate and need for mechanical ventilation in COVID19 patients, while insignificant differences in risk of infection and other safety outcomes were seen between 
the experimental and control group. Secondly, there was a significant improvement in the efficacy outcome, i.e., improved oxygen saturation, in the group treated with tocilizumab.

Evidence from multiple studies suggests a strong role of Cytokine Release Syndrome (CRS) in the development of ARDS and lung failure [23-26]. A meta-analysis by Coomes et al. revealed a 2.9 times higher mean IL-6 concentration in complicated COVID-19 cases versus non-complicated ones [27]. Since the key cytokine in this hyper-inflammatory response is IL-6, it seems imperative to test the efficacy of IL-6 blockers such as tocilizumab in treating critical COVID-19 patients [24-26]. Tocilizumab is approved by the FDA to treat rheumatoid arthritis, giant cell arteritis, poly-articular juvenile idiopathic arthritis, systemic juvenile idiopathic arthritis, and now CAR-T cell-induced CRS [23-25]. It has proven efficacy in attenuating inflammation and has a well-known long-term safety profile, which makes it suitable for use as an adjunctive immune modulator along with other therapies [23-26].

The baseline characteristics and outcomes of six single-arm, observational studies investigating the safety of tocilizumab therapy in COVID-19 patients are shown in Table 7 [17-22]. All studies show a significantly lower all-cause mortality than that seen in other critically ill COVID-19 cohorts. Almost all the studies show a rapid decline in fever and laboratory markers of inflammation, while there is a statistically significant improvement in subsequent CT scan imaging and oxygen requirements $[4,5,17]$. This evidence strongly suggests the efficacy of tocilizumab not as a standalone COVID-19 therapy but as an effective adjunct treatment nonetheless, in the subgroup of COVID-19 patients who develop CRS. However, the reliability of these results is limited by their retrospective nature, lack of a control arm for comparison of results, and lack of a defined treatment protocol. That increases the need for other better-designed studies, preferably RCTs.

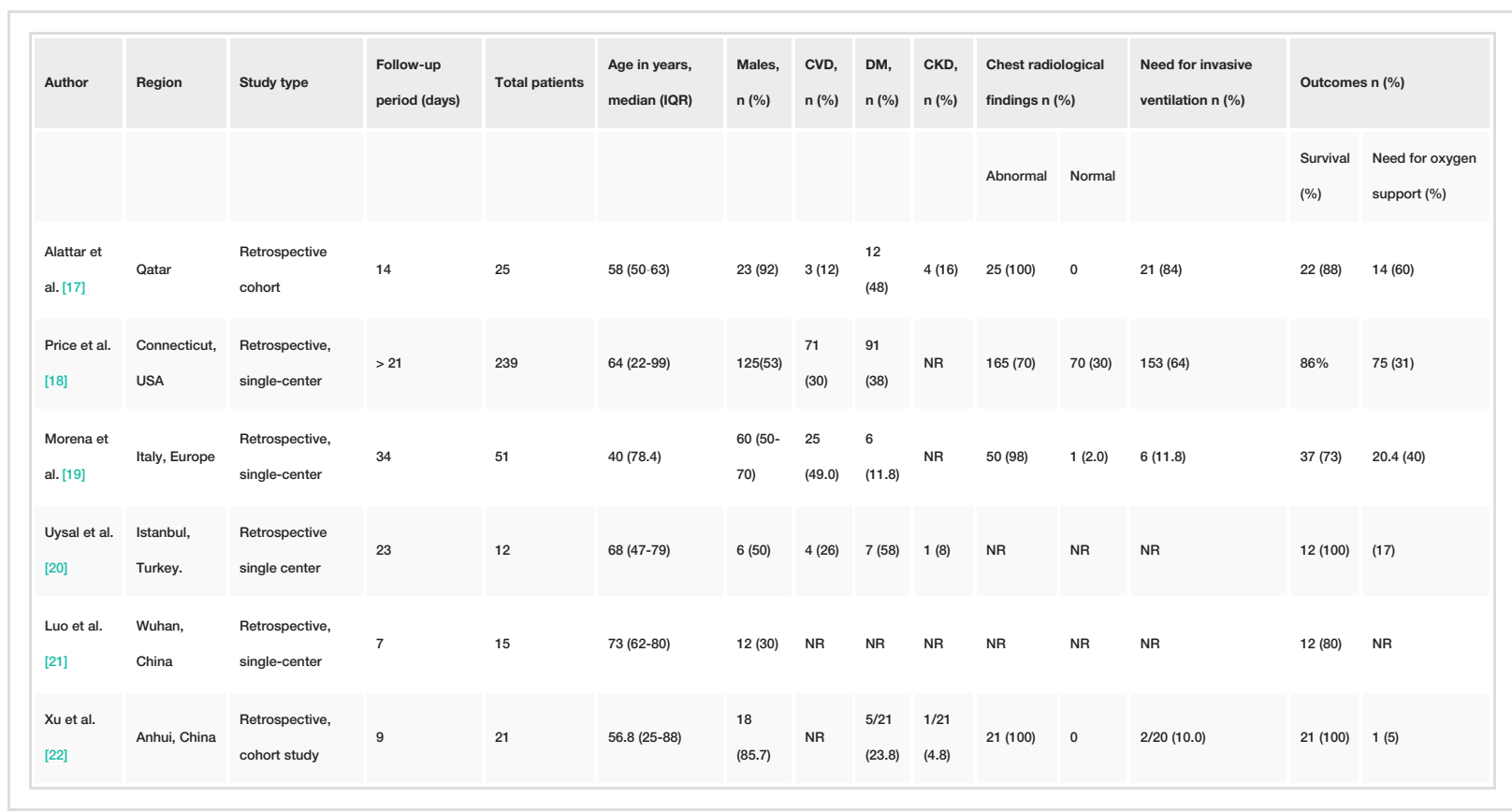

TABLE 7: Baseline characteristics and outcomes of single-arm studies

CVD, Cardiovascular disease; DM, Diabetes mellitus; CKD, Chronic kidney disease; IQR, interquartile range, NR, not reported

The meta-analysis suggests a significantly lower risk of mortality among the patients who received tocilizumab therapy along with standard therapy compared to those receiving standard therapy alone. This finding is consistent with the findings of most comparative observational studies, including those by Klopfenstein et al. and Capra et al. [10-11]. In contrast to this, Rojas-Marte et al. reported no significant difference in mortality between the two groups; however, the difference was significant once already intubated patients were excluded [12]. Similarly, in the study by Quartico et al., among the subgroup of patients on a ventilator in the tocilizumab-treated group, approximately $50 \%$ did not respond to treatment [13]. Furthermore, the meta-analysis showed no significant difference in ICU admissions. These findings strongly suggest that tocilizumab may perhaps be more effective in controlling the early stages of the inflammation and cannot reverse the hyper-inflammatory state once reached. Conversely, the COVACTA trial, which is the first global, randomized, double-blind, placebo-controlled phase III study evaluating the safety and efficacy of tocilizumab in the patients with severe COVID-19 pneumonia, shows no difference in patient mortality at week four [6].

Consistent with the results of the single-arm observational studies, this meta-analysis also demonstrated 
that a significantly lower number of patients requiring mechanical ventilation following tocilizumab therapy and a significant improvement in their blood oxygen saturation [4-5,26]. This is an important finding in the context of the low availability and high costs of ventilators. Tocilizumab therapy seems to be beneficial in relieving the tremendous economic burden on public health systems.

Tocilizumab is known to pose an increased risk of secondary bacterial/fungal infections due to its immunosuppressive mode of action. However, the pooling of the results demonstrated no significant difference in infections between the two groups. One plausible explanation may be the lower rate of intubation and mechanical ventilation in these patients, eliminating risk from infection-causing procedures. Interestingly, scientists in favor of tocilizumab suggest that the desquamation of alveolar cells, hyaline membrane formation, and pulmonary edema following the hyper-inflammatory state make the lungs more vulnerable to secondary infections, and hence tocilizumab has a protective action [24]. Also, it is noteworthy that tocilizumab is known to cause infections only on continuous dosing, whereas the maximum number of doses administered to COVID-19 patients in these trials is two [26]. Besides, this analysis shows no significant difference in the advent of serious adverse events (SAE) among the two groups. This indicates a low risk-benefit ratio favoring the use of tocilizumab in critically ill COVID-19 patients [23].

Patients were administered corticosteroid therapy as part of the standard regimen in five of the seven cohorts. Corticosteroids have a potent anti-inflammatory effect [28]. The possible overlap of its effects with those of tocilizumab generates significant confounding. The results of the subgroup analysis reported no significant difference in safety and efficacy outcomes except significantly reduced infections in the corticosteroid subgroup (Table 4). Corticosteroids are known to cause immunosuppression and possibly increase the risk of infection when administered in very high doses ( $>100 \mathrm{mg} /$ day) over an extended period [29]. However, the studies included in this analysis that employ the use of corticosteroid therapy had administered moderate doses of corticosteroids for no more than 10 days; therefore, they pose no increased risk of infections. On the contrary, they may reduce the incidence of post-infectious inflammation.

\section{Limitations}

There are several limitations that this meta-analysis attempts to overcome, including the limited sample sizes, non-homogenous sample population, and short duration of studies. To minimize inter-study heterogeneity, the baseline characteristics of both cohorts were adjusted. However, the lack of a uniform standard protocol for treatment and no clear guidelines on the dosing and administration of tocilizumab generate a confounding bias. Moreover, the populations differed in disease severity and the relative time of treatment during the course of the disease.

Tocilizumab appears to improve mortality and oxygen saturation as compared to controls upon pooling of the observational studies; however, the COVACTA trial showed no mortality benefit [6]. The observational studies were of low quality and mostly unadjusted; thus, the mortality benefit seen in these studies may be spurious and due to confounding. On the contrary, the COVACTA trial had a small sample size and low power [6]. Thus, current findings should be viewed cautiously, as neither the RCT nor the meta-analysis of observational studies shows much improvement in the safety outcome, so it is questionable as to where the mortality benefit came from.

\section{Conclusions}

This meta-analysis shows that therapy with tocilizumab lowers the mortality rate and risk of artificial ventilation in COVID-19 patients and improves their oxygen saturation levels. While it has no role in reducing ICU admissions, it does not pose a serious risk of infections and other adverse events. Furthermore, concomitant corticosteroid therapy seems to lower the risk of infections. Similarly, the six single-arm studies show a lower mortality rate with tocilizumab therapy. Tocilizumab is a promising agent to attenuate inflammation and cytokine release, which is the underlying pathophysiology of several COVID-19 cases. However, the poor design and various limitations of the studies render them ineffective in gauging the full extent of its safety and efficacy and thus warrant further research into the use of tocilizumab.

\section{Additional Information \\ Disclosures}

Human subjects: All authors have confirmed that this study did not involve human participants or tissue. Animal subjects: All authors have confirmed that this study did not involve animal subjects or tissue. Conflicts of interest: In compliance with the ICMJE uniform disclosure form, all authors declare the following: Payment/services info: All authors have declared that no financial support was received from any organization for the submitted work. Financial relationships: All authors have declared that they have no financial relationships at present or within the previous three years with any organizations that might have an interest in the submitted work. Other relationships: All authors have declared that there are no other relationships or activities that could appear to have influenced the submitted work.

\section{References}


1. Rothan HA, Byrareddy SN: The epidemiology and pathogenesis of coronavirus disease (COVID-19) outbreak . J Autoimmun. 2020, 109:102433. 10.1016/j.jaut.2020.102433

2. Chan JF, Zhang AJ, Yuan S, et al.: Simulation of the clinical and pathological manifestations of coronavirus disease 2019 (COVID-19) in golden Syrian hamster model: implications for disease pathogenesis and transmissibility. Clin Infect Dis. 2020, [Epub ahead of print]:ciaa325. 10.1093/cid/ciaa325

3. Ulhaq ZS, Soraya GV: Interleukin-6 as a potential biomarker of COVID-19 progression . Med Mal Infect. 2020, 50:382-383. 10.1016/j.medmal.2020.04.002

4. Biran N, Ip A, Ahn J, et al.: Tocilizumab among patients with COVID-19 in the intensive care unit: a multicentre observational study. Lancet Rheumatol. 2020, 2:603-612. 10.1016/S2665-9913(20)30277-0

5. Luo P, Liu Y, Qiu L, Liu X, Liu D, Li J: Tocilizumab treatment in COVID- 19: a single center experience . J Med Virol. 2020, 92:814-818. 10.1002/jmv.25801

6. Roche provides an update on the phase III COVACTA trial of Actemra/RoActemra in hospitalised patients with severe COVID-19 associated pneumonia. (2020). Accessed: August 12, 2020: https://www.roche.com/investors/updates/inv-update-2020-07-29.htm.

7. Hutton B, Salanti G, Caldwell DM, et al.: The PRISMA extension statement for reporting of systematic reviews incorporating network meta-analyses of health care interventions: checklist and explanations. Ann Intern Med. 2015, 162:777-784. 10.7326/M14-2385

8. Higgins JP, Altman DG, Gøtzsche PC, et al.: The Cochrane Collaboration's tool for assessing risk of bias in randomised trials. BMJ. 2011, 343:d5928. 10.1136/bmj.d5928

9. The Newcastle-Ottawa Scale (NOS) for assessing the quality of nonrandomised studies in meta-analyses . (2019). Accessed: August 12, 2020: http://www.ohri.ca/programs/clinical_epidemiology/oxford.asp.

10. Klopfenstein T, Zayet S, Lohse A, et al.: Tocilizumab therapy reduced intensive care unit admissions and/or mortality in COVID-19 patients. Med Mal Infect. 2020, 50:397-400. 10.1016/j.medmal.2020.05.001

11. Capra R, De Rossi N, Mattioli F, Romanelli G, Scarpazza C, Sormani MP, Cossi S: Impact of low dose tocilizumab on mortality rate in patients with COVID-19 related pneumonia. Eur J Intern Med. 2020, 76:3135. 10.1016/j.ejim.2020.05.009

12. Rojas-Marte G, Khalid M, Mukhtar O, et al.: Outcomes in patients with severe COVID-19 disease treated with tocilizumab: a case-controlled study. QJM. 2020, 113:546-550. 10.1093/qjmed/hcaa206

13. Quartuccio L, Sonaglia A, McGonagle D, et al.: Profiling COVID-19 pneumonia progressing into the cytokine storm syndrome: results from a single Italian centre study on tocilizumab versus standard of care. J Clin Virol. 2020, 129:104444. 10.1016/j.jcv.2020.104444

14. Somers EC, Eschenauer GA, Troost JP, et al.: Tocilizumab for treatment of mechanically ventilated patients with COVID-19 [Preprint]. medRxiv. 2020, 10.1101/2020.05.29.20117358

15. Colaneri M, Bogliolo L, Valsecchi P, et al.: Tocilizumab for Treatment of severe COVID-19 patients: preliminary results from SMAtteo COvid19 REgistry (SMACORE). Microorganisms. 2020, 9:695. 10.3390/microorganisms8050695

16. Campochiaro C, Della-Torre E, Cavalli G, et al.: Efficacy and safety of tocilizumab in severe COVID-19 patients: a single-centre retrospective cohort study. Eur J Intern Med. 2020, 76:43-49. 10.1016/j.ejim.2020.05.021

17. Alattar R, Ibrahim TBH, Shaar SH, et al.: Tocilizumab for the treatment of severe coronavirus disease 2019 . J Med Virol. 2020, 92:2042-2049. 10.1002/jmv.25964

18. Price CC, Altice FL, Shyr Y, et al.: Tocilizumab treatment for cytokine release syndrome in hospitalized COVID-19 patients: survival and clinical outcomes [Epub ahead of print]. Chest. 2020, 158:1397-1408. 10.1016/j.chest.2020.06.006

19. Morena V, Milazzo L, Oreni L, et al.: Off-label use of tocilizumab for the treatment of SARS-CoV-2 pneumonia in Milan, Italy. Eur J Intern Med. 2020, 76:36-42. 10.1016/j.ejim.2020.05.011

20. Borku-Uysal B, Ikitimur H, Yavuzer S, et al.: Tocilizumab challenge: a series of cytokine storm therapy experiences in hospitalized COVID-19 pneumonia patients. J Med Virol. 2020, 92:2648-2656. 10.1002/jmv.26111

21. Luo P, Liu Y, Qiu L, Liu X, Liu D, Li J: Tocilizumab treatment in COVID- 19: a single center experience . J Med Virol. 2020, 92:814-818. 10.1002/jmv.25801

22. Xu X, Han M, Li T, et al.: Effective treatment of severe COVID-19 patients with tocilizumab . Proc Natl Acad Sci U S A. 2020, 117:10970-10975. 10.1073/pnas.2005615117

23. Mehta P, McAuley DF, Brown M, Sanchez E, Tattersall RS, Manson JJ, HLH Across Speciality Collaboration UK: COVID- 19: consider cytokine storm syndromes and immunosuppression . Lancet. 2020, 395:1033-1034. 10.1016/S0140-6736(20)30628-0

24. Atal S, Fatima Z: IL-6 Inhibitors in the treatment of serious COVID- 19: a promising therapy? . Pharmaceut Med. 2020, 34:223-231. 10.1007/s40290-020-00342-Z

25. Zhang S, Li L, Shen A, Chen Y, Qi Z: Rational use of tocilizumab in the treatment of novel coronavirus pneumonia. Clin Drug Investig. 2020, 40:511-518. 10.1007/s40261-020-00917-3

26. Fu B, Xu X, Wei H: Why tocilizumab could be an effective treatment for severe COVID-19? . J Transl Med. 2020, 18:164. 10.1186/s12967-020-02339-3

27. Coomes EA, Haghbayan H: Interleukin-6 in COVID- 19: a systematic review and meta-analysis . Rev Med Virol. 2020, [Epub ahead of print]:e2141. 10.1002/rmv.2141

28. Youssef J, Novosad SA, Winthrop KL: Infection risk and safety of corticosteroid use . Rheum Dis Clin North Am. 2016, 42:157-176.

29. Gensler LS: Glucocorticoids: complications to anticipate and prevent. Neurohospitalist. 2013, 3:92-97. $10.1177 / 1941874412458678$ 